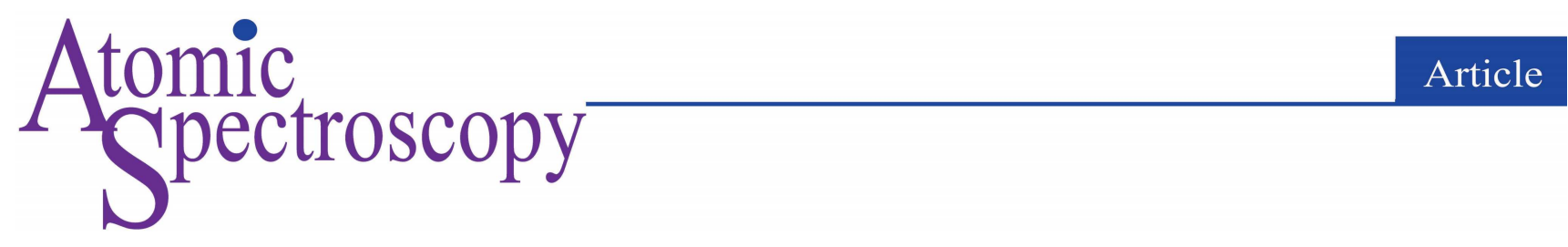

\title{
Determining Trace Rare-earth Oxides in High-purity Europium Oxide by Microsecond-pulsed and Continuous Direct-current Glow- discharge Mass Spectrometry
}

\author{
Jianying Zhang, Tao Zhou,* Yanjie Cui, Yichuan Tang, and Bing Wu \\ National Institute of Metrology, Beijing, 100029, P.R. China
}

Received: December 18, 2020; Revised: January 06, 2021; Accepted: January 06, 2021; Available online: January $19,2021$.

DOI: $10.46770 / A S .2020 .214$

ABSTRACT: Establishing the purities of rare-earth oxides is an important objective as they are extensively used in a variety of applications. Herein, we reported the development of mixing and secondarycathode methods for the microsecond-pulsed and continuous direct-current glowdischarge mass spectrometric analyses of trace rare-earth-oxide contaminants present in europium oxide samples. Matrix effects were corrected for, results were calibrated using relative sensitivity factors derived from pure copper, and the accuracy of the method was validated using $\mathrm{Eu}_{2} \mathrm{O}_{3}$ certified reference materials, with relative deviations of less than $15 \%$ obtained for most analytes in both modes.
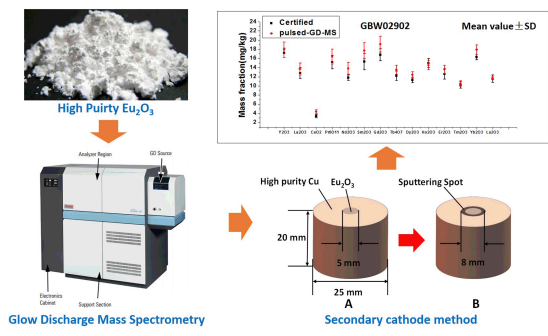

\section{INTRODUCTION}

High-purity rare-earth oxides, such as europium oxide $\left(\mathrm{Eu}_{2} \mathrm{O}_{3}\right)$, are extensively employed as advanced ceramic materials and hydrogenous storage materials, ${ }^{1}$ among others. The purity requirements of rare-earth oxides are specified in the first version of the "Catalogue of Demonstration Guide for Key New Materials" released by the Ministry of Industry and Information Technology of P. R. China. The mass fractions of rare-earth elements need to be known because they can considerably affect the properties of the material. Inductively coupled plasma mass spectrometry (ICPMS) is the most common technique used to analyze trace rareearth elements in high-purity rare-earth oxides. ${ }^{2,3}$ However, the required sample dissolution and separation steps are very timeconsuming, while dilution and the use of high blank reagents can negatively affect detection limits. In addition, as a solutionsampling technique, complex hydrogen- and oxygen-based polyatomic spectral interference can severely hamper the analysis of some rare-earth elements; i.e., ${ }^{165} \mathrm{Ho}^{+}$is interfered with by ${ }^{164} \mathrm{DyH}^{+},{ }^{141} \mathrm{Pr}^{+}$is interfered with by ${ }^{140} \mathrm{CeH}^{+}$, and ${ }^{169} \mathrm{Tm}^{+}$is interfered with by ${ }^{168} \mathrm{ErH}^{+}$and ${ }^{153} \mathrm{Eu}^{16} \mathrm{O}^{+}$. Therefore, a rapid, sensitive, and reliable analytical method for the analysis of trace rare-earth oxides (elements) in high-purity rare-earth oxides is of urgent need.
As a solid sampling technique, glow-discharge mass spectrometry (GD-MS) is a powerful tool for analyzing trace elements in high-purity materials; ${ }^{4,5}$ further, its simple sample pretreatment protocol significantly improves analysis efficiency. High sensitivities $(\sim 100 \mathrm{cps} / \mathrm{ppb})$ result in very low detection limits (down to $\mathrm{ng} \mathrm{kg}^{-1}$ ) in solid materials. Moreover, the polyatomic interferences derived from hydrogen and oxygen are very low because a dry aerosol is used. In addition, the use of the high-resolution $(m / \Delta m>10000)$ mode eliminates most spectral interference. Therefore, GD-MS is well-suited to the analysis of trace rare-earth elements. Direct-current glow-discharge (dc-GD) and radio frequency glow-discharge (rf-GD) sources are the two commonly used GD sources, ${ }^{6}$ and both can be used in the continuous and pulsed modes.

The sample needs to be electrically conducting in the dc-GD system, as it serves as the cathode. Therefore, a challenge of this work involves preparing electrically conducting samples. Auxiliary conductors, such as high-purity metal powder or foil, are necessary when analyzing non-conductive materials. A secondarycathode technique can be used for pin-shaped ${ }^{7,8}$ or plate-shaped ${ }^{9,10}$ solid samples. On the other hand, mixing with a high-purity conducting powder is always required for powdered samples, ${ }^{11,12}$ although the mixing procedure may lead to some elemental 
contamination.

In addition to problems associated with sample conductivity, accurate quantitative analysis is also challenging, due to a lack of matrix-matched certified reference materials (CRMs) with known chemical compositions similar to that of the sample to be analyzed. Generally, standard relative sensitivity factors (StdRSFs), ${ }^{13}$ which are matrix-independent, are used to calibrate the acquired data. However, the use of StdRSFs still leads to relatively large uncertainties (of a factor of approximately two) ${ }^{14,15}$ in most cases, and matrix-matched RSFs are always needed to provide more accurate results. Synthetic pressed tablets ${ }^{5,16}$ and melting bulks ${ }^{15}$ are two commonly used types of metal calibration sample; however, calibration samples of melting bulks and pressed tablets of europium oxides are difficult to prepare because auxiliary conductors are required.

In this work, europium oxides were examined by microsecondpulsed and continuous dc GD-MS, and mixing and secondarycathode methods were established. Matrix effects were corrected for in order to calibrate the results using RSFs derived from pure copper, and the accuracy of the method was validated using $\mathrm{Eu}_{2} \mathrm{O}_{3}$ CRMs. Finally, a real europium oxide sample was examined and its relative purity determined.

\section{EXPERIMENTAL}

Mass spectrometer. An Element GD Plus (Thermo Fisher Scientific, USA) high-resolution double-focusing sector-field glow-discharge mass spectrometer, equipped with a fast-flow Grimm-type direct-current (dc) ion source, was used in this study. The ion source can be operated in both microsecond-pulsed and continuous modes. The instrument has three mass-resolution modes, namely low (LR), medium (MR), and high resolution (HR), with resolution values of approximately 400, 4000, and 10000 , respectively. The sample is required to be electrically conducting in the ion source because the sample serves as the cathode in the GD system.

Reagents and samples. High-purity 22-mesh copper powder with a nominal purity of $99.999 \%$ was purchased from Alfa Aesar Corp. (Ward Hill, MA, USA). The powder was sieved in-house into two size ranges. The powder with grains $500-600 \mu \mathrm{m}$ in size was used to prepare the calibration samples needed to calculate the RSFs of the rare-earth elements. The powder with grains less than $150 \mu \mathrm{m}$ in size was used as the auxiliary conductor when preparing electrically conducting samples for GD-MS. Multi-element standard solutions of 17 rare-earth elements with mass fractions of $100 \mathrm{mg} / \mathrm{kg}$ were provided by the National Institute of Metrology (NIM), P.R. China. The impurity constituents of GBW02902 europium oxide CRMs were used for validation purposes. A sample of high-purity $\mathrm{Eu}_{2} \mathrm{O}_{3}$ with a nominal purity of more than 99.999\% was purchased from Alfa Aesar (Tianjing, China).
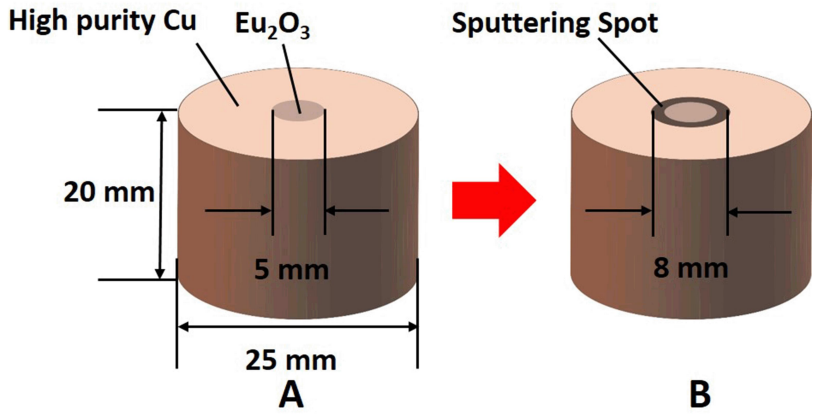

Fig. 1 High-purity copper secondary cathode (A) before and (B) after GDMS sputtering.

Table 1. Optimized Values of the Discharge Parameters Used in Directcurrent GD-MS

\begin{tabular}{lcc}
\hline Discharge Parameter & $\begin{array}{c}\text { Microsecond- } \\
\text { pulsed }\end{array}$ & Continuous \\
\hline Discharge voltage (V) & 1000 & $1200 \mathrm{~V}$ \\
Discharge current (mA) & 30 & 55 \\
Discharge gas flow (mL & 505 & 430 \\
min $\left.^{-1}\right)$ & & \\
Pulsed duration ( $\boldsymbol{\mu s})$ & 70 & $\mathrm{ND}^{[\mathrm{a}]}$ \\
Pulsed frequency (Hz) & 2000 & $\mathrm{ND}^{[\mathrm{a}]}$ \\
\hline
\end{tabular}

${ }^{[a]}$ No data.

Sample preparation. The calibration samples used to determine RSFs were prepared by doping high-purity copper powder with rare-earth standard solutions. Details of the preparation procedure are reported elsewhere., ${ }^{5,15}$ The mixing and secondary-cathode methods were used to directly analyze the non-conducting $\mathrm{Eu}_{2} \mathrm{O}_{3}$. In the mixing method, mixtures, each with an optimal $\mathrm{Cu} / \mathrm{Eu}_{2} \mathrm{O}_{3}$ mass ratio of 4:1, were prepared by mechanical mixing. Tablets of the mixtures were prepared by pressing each homogenized powder in a filling ring (i.d. $=12 \mathrm{~mm}$; o.d. $=25 \mathrm{~mm}$; height $=1.2 \mathrm{~mm}$ ) using a pressing machine (SYP-30TS, Shanghai, China) with a pressure of $30 \mathrm{MPa}$ for a hold time of $20 \mathrm{~s}$. In the secondarycathode method, a 20 mm-thick cylindrical high-purity (>99.999\%) copper block with a $5 \mathrm{~mm}$-diameter hole was custommade by China New Metal Co., Ltd. (Beijing, China). The surface was cleaned by grinding with a lapping machine (LaboPol-5, Struers, USA) and then ultrasonically cleaned using deionized water and ethanol. The $\mathrm{Eu}_{2} \mathrm{O}_{3}$ sample was loaded by pressing at $0.5 \mathrm{MPa}$ with a pressure lever for a hold time of approximately 20 $\mathrm{s}$, as shown in Fig. 1.

Glow-discharge mass spectrometry. Based on a previous study, ${ }^{17}$ GD-MS was performed in the microsecond-pulsed and dc modes when the mixing method was used, while only the microsecond-pulsed mode was used with the secondary-cathode method. The discharge parameters were optimized for high intensity and sufficient ${ }^{151} \mathrm{Eu}$ analytical stability. The optimal values of the discharge parameters in the microsecond-pulsed and continuous dc modes are summarized in Table 1. Each experiment was carried out six times in succession, with the average value 


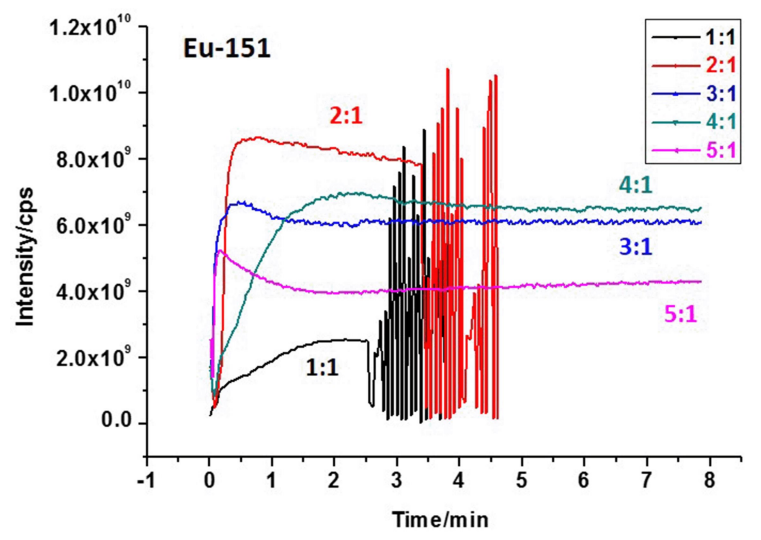

Fig. 2 Mixing method: ${ }^{151} \mathrm{Eu}$ signal intensity at medium resolution (MR) as functions of time and the mass ratio of high-purity $\mathrm{Cu}$ to $\mathrm{Eu}_{2} \mathrm{O}_{3}$ in the continuous de mode.

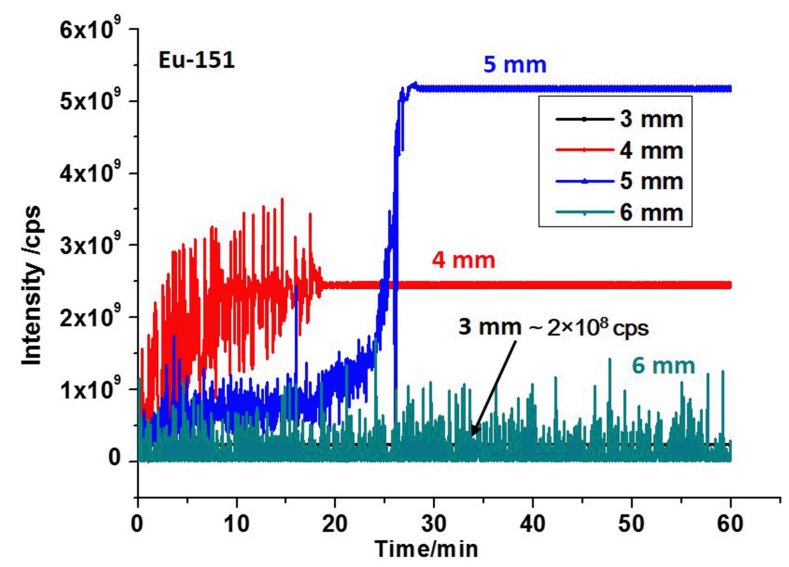

Fig. 3 Secondary-cathode method: ${ }^{151}$ Eu signal intensity at medium resolution (MR) as functions of time and the secondary cathode hole diameter in the microsecond-pulsed mode.

Table 2. Limits of Quantification (LoQ) of Rare-earth Oxides Using the Mixing and Secondary-cathode Methods

\begin{tabular}{|c|c|c|c|c|c|}
\hline \multirow[b]{2}{*}{ Oxide } & \multicolumn{2}{|c|}{ LoQ, mg/kg } & \multirow[b]{2}{*}{ Oxide } & \multicolumn{2}{|c|}{ LoQ, mg/kg } \\
\hline & Mixing & $\begin{array}{l}\text { Secondary } \\
\text { Cathode }\end{array}$ & & Mixing & $\begin{array}{c}\text { Secondary } \\
\text { Cathode }\end{array}$ \\
\hline $\mathrm{Y}_{2} \mathrm{O}_{3}$ & 0.17 & 0.005 & $\mathbf{T b}_{4} \mathbf{O}_{7}$ & 0.002 & 0.001 \\
\hline $\mathbf{L a}_{2} \mathrm{O}_{3}$ & 0.012 & 0.002 & $\mathrm{Dy}_{2} \mathrm{O}_{3}$ & 0.001 & 0.001 \\
\hline $\mathrm{CeO}_{2}$ & 0.79 & 0.041 & $\mathrm{Ho}_{2} \mathrm{O}_{3}$ & 0.012 & 0.004 \\
\hline $\operatorname{Pr}_{6} \mathbf{O}_{11}$ & 0.081 & 0.002 & $\mathrm{Er}_{2} \mathrm{O}_{3}$ & 0.007 & 0.001 \\
\hline $\mathrm{Nd}_{2} \mathrm{O}_{3}$ & 0.35 & 0.016 & $\mathbf{T m}_{2} \mathbf{O}_{3}$ & 0.003 & 0.001 \\
\hline $\mathrm{Sm}_{2} \mathrm{O}_{3}$ & 0.11 & 0.020 & $\mathbf{Y} \mathbf{b}_{2} \mathbf{O}_{3}$ & 0.007 & 0.019 \\
\hline $\mathbf{G d}_{2} \mathrm{O}_{3}$ & 0.030 & 0.022 & $\mathrm{Lu}_{2} \mathrm{O}_{3}$ & 0.001 & 0.001 \\
\hline SUM & 1.6 & 0.14 & & & \\
\hline
\end{tabular}

reported. The mass fraction of each rare-earth element was calculated using the following equation:

$$
\frac{\mathrm{C}_{x}}{\mathrm{C}_{\mathrm{Eu}_{2} \mathrm{O}_{3}}}=\left(\frac{\mathrm{I}_{x}}{\mathrm{I}_{\mathrm{Eu}}}-\frac{\mathrm{I}_{x}}{\mathrm{I}_{\mathrm{Cu}}} \cdot \mathrm{R}\right) \times \frac{\mathrm{RSF}_{\mathrm{x} / \mathrm{Cu}}}{\mathrm{RSF}_{\mathrm{Eu} / \mathrm{Cu}}} \cdot \frac{\mathrm{M}_{\mathrm{Eu}}}{\mathrm{M}_{\mathrm{Eu}_{2} \mathrm{O}_{3}}}
$$

where $\mathrm{C}_{x} / \mathrm{C}_{\mathrm{Eu} 2 \mathrm{O} 3}$ is the mass fraction of the element in $\mathrm{Eu}_{2} \mathrm{O}_{3}$; $\mathrm{I}_{x} / \mathrm{I}_{\mathrm{Eu}}, \mathrm{I}_{x} / \mathrm{I}_{\mathrm{Cu}}$, and $\mathrm{R}$ are the ion beam ratio (IBR) of element $x$ to Eu derived from the mixture, the IBR of element $x$ to $\mathrm{Cu}$ derived from the auxiliary $\mathrm{Cu}$ conductor, and the IBR of $\mathrm{Cu}$ to $\mathrm{Eu}$ from the mixture, respectively; and $\mathrm{M}$ is the molecular/atomic weight. The mass fraction of element $x$ is multiplied by the ratio of the molecular mass of the oxide to the element, in the case of an oxide.

\section{RESULTS AND DISCUSSION}

Optimizing the mass ratio in the mixing method and the hole diameter in the secondary-cathode method. The mass ratio of high-purity copper powder to $\mathrm{Eu}_{2} \mathrm{O}_{3}$ was optimized to obtain high sensitivity and good stability in the mixing method; the intensities of the ${ }^{151} \mathrm{Eu}$ signals from 1:1, 2:1, 3:1, 4:1, and 5:1 mixture at MR (Fig. 2). In view of both sensitivity and stability, a 4:1 Cu/ $\mathrm{Eu}_{2} \mathrm{O}_{3}$ mass ratio was determined to be optimal, with a ${ }^{151} \mathrm{Eu}$ sensitivity of approximately $7 \times 10^{9} \mathrm{cps}$ and a relative standard deviation (RSD) of less than $1 \%$. The diameter of the hole was also optimized in the secondary-cathode method by comparing the intensity, ${ }^{151} \mathrm{Eu}$ stability, and equilibrium time, as shown in Fig. 3. More of the $\mathrm{Eu}_{2} \mathrm{O}_{3}$ sample is exposed to the GD ion source with increasing hole diameter, resulting in a more-intense ${ }^{151} \mathrm{Eu}$ signal; however, less of the $\mathrm{Cu}$ conductor is exposed to the GD ion source, which increases the equilibrium time. A diameter of $5 \mathrm{~mm}$ was determined to be optimal, with a ${ }^{151}$ Eu sensitivity of approximately $5 \times 10^{9} \mathrm{cps}$, an RSD of less than $1 \%$, and an equilibrium time of approximately $25 \mathrm{~min}$. Even though the equilibrium time associated with the secondary cathode with the $5 \mathrm{~mm}$ hole was much longer than that with the $3 \mathrm{~mm}$ hole (approximately 2-3 $\mathrm{min}$ ), the intensity of the ${ }^{151} \mathrm{Eu}$ signal was more than one order of magnitude higher that that observed with the $3 \mathrm{~mm}$ hole, for which the sensitivity was approximately $2 \times 10^{8} \mathrm{cps}$.

Limits of quantification (LoQ) of rare-earth oxides determined by the mixing and secondary-cathode methods. The LoQs of rare-earth oxides were determined statistically for the mixing and secondary-cathode methods using the 10 s-criteron, as summarized in Table 2. The LoQ of most oxides were approximately one order of magnitude lower using the secondarycathode method compared to those obtained using the mixing method, due to the lower blanks in the high-purity copper secondary cathode. The mass fractions of most rare-earth elements were less than $0.003 \mathrm{mg} / \mathrm{kg}$ in the high-purity copper secondary cathode, with the exception of Ce (0.005), Gd (0.004), Sm (0.003), and $\mathrm{Yb}(0.003)$. The LoQ across all oxides were $1.6 \mathrm{mg} / \mathrm{kg}$ and $0.14 \mathrm{mg} / \mathrm{kg}$ for the mixing and secondary-cathode methods, respectively. Hence, europium oxides with relative purities of more than $99.999 \%$ and $99.9999 \%$ can be measured and identified by the mixing and secondary-cathode methods, respectively.

Matrix effects in microsecond-pulsed and continuous dc GDMS. In order to better calibrate the acquired data using the RSFs derived from pure $\mathrm{Cu}$, which are relatively easy to obtain, the effects of the GD-MS matrix in the microsecond-pulsed and 


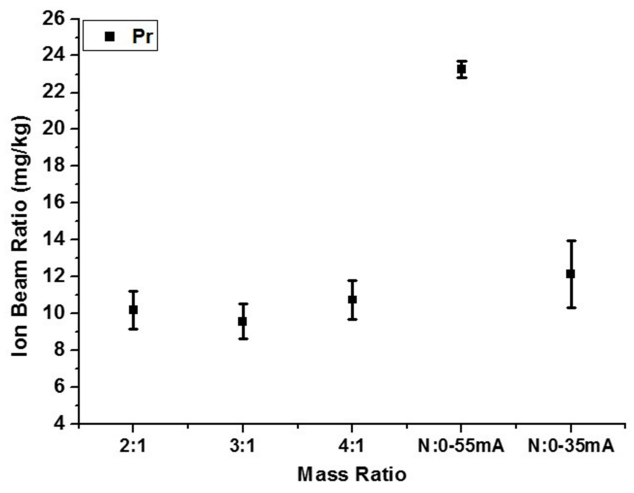

Fig. 4 Matrix effects in the continuous dc mode: Pr-to-Cu ion beam ratios (IBRs) of different mixtures of doped copper (high-purity copper doped with $10 \mathrm{mg} / \mathrm{kg}$ rare-earth standard solutions) in high-purity $\mathrm{Eu}_{2} \mathrm{O}_{3}$ in the continuous dc mode at medium resolution (MR). Error bars represent standard deviations (SDs; $\mathrm{n}=6$ ).

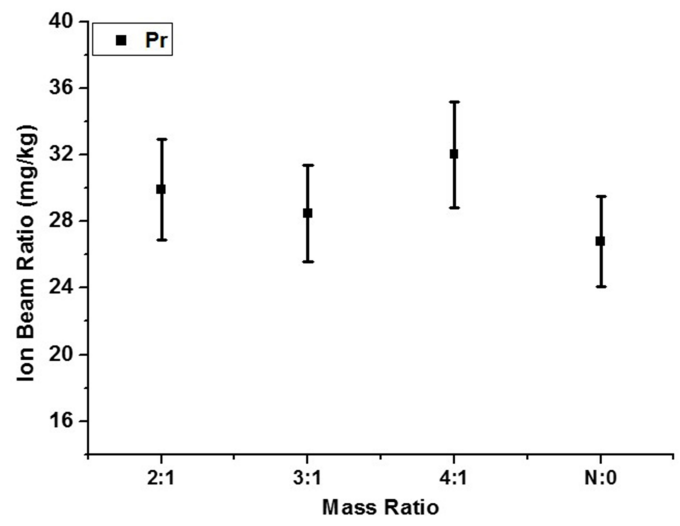

Fig. 5 Matrix effects in the microsecond-pulsed mode: Pr-to-Cu ion beam ratios (IBRs) of different mixtures of doped copper (high-purity copper doped with $10 \mathrm{mg} / \mathrm{kg}$ rare-earth standard solutions) in high-purity $\mathrm{Eu}_{2} \mathrm{O}_{3}$ in the microsecond-pulsed mode at medium resolution (MR). Error bars represent standard deviations (SDs; $\mathrm{n}=6$ ).

continuous de modes were investigated. High-purity $\mathrm{Cu}$ powder samples doped with $10 \mathrm{mg} / \mathrm{kg}$ elemental rare-earth standard solutions were prepared and are referred to as "doped copper" powder in this work. High-purity $\mathrm{Eu}_{2} \mathrm{O}_{3}$, for which the mass fractions of all rare-earth elements were very low $(<0.1 \mathrm{mg} / \mathrm{kg})$, was used. Mixtures of "doped copper" and high-purity $\mathrm{Eu}_{2} \mathrm{O}_{3}$ with mass ratios of $2: 1,3: 1,4: 1$, and $\mathrm{N}: 0$ (doped $\mathrm{Cu}$ only) were prepared. The rare-earth element in each of the various mixtures had the same mass fraction relative to $\mathrm{Cu}$ (the rare-earth elements in $\mathrm{Eu}_{2} \mathrm{O}_{3}$ can be ignored); hence the matrix effect can be studied by comparing the IBRs of the rare-earth elements relative to $\mathrm{Cu}$.

An optimized discharge current of $55 \mathrm{~mA}$ was used in the continuous dc mode when the $2: 1,3: 1$, and $4: 1$ mixture were examined, with a ${ }^{63} \mathrm{Cu}$ sensitivity of approximately $7 \times 10^{9} \mathrm{cps}$ determined at MR for the 4:1 mixture. The Pr-to-Cu IBRs, as examples, in the various mixtures are compared in Fig. 4, which shows that the IBR of Pr in the N:0 sample at $55 \mathrm{~mA}$ is twice that of the 4:1 mixture, thereby confirming the presence of a marked matrix effect. This observation is attributable to variations in the real-time discharge voltage, which was $589 \mathrm{~V}$ at $55 \mathrm{~mA}$ when analyzing the $4: 1$ mixture, but $1081 \mathrm{~V}$ when analyzing the $\mathrm{N}: 0$ sample. The Pr sputtering efficiency is enhanced at a higher discharge voltage, leading to a distinct IBR discrepancy. The results obtained for the mixtures were calibrated using the RSFs determined using the $\mathrm{N}: 0$ calibration sample at a current of $35 \mathrm{~mA}$. The IBRs for Pr in the 4:1 mixture and the N:0 sample agree well at $35 \mathrm{~mA}$. The real-time voltage and current were approximately $800 \mathrm{~V}$ and $33.6 \mathrm{~mA}$, respectively, at $35 \mathrm{~mA}$; the voltage was higher and the current was lower than those observed at $55 \mathrm{~mA}$ when the 4:1 mixture was analyzed. The opposite effect resulted in good agreement. In other words, the matrix effect was corrected for by optimizing the discharge parameters. Accurate values can be obtained in the dc mode when calibrated using RSFs measured at $35 \mathrm{~mA}$ (other parameters listed in Table 1) using the N:0 sample.

The same discharge parameters were used to analyze the various mixtures in the pulsed mode. The Pr-to-Cu IBRs were in good agreement to within uncertainty intervals, even though the IBR of the $4: 1$ sample appeared to be $15 \%$ higher than that of the $\mathrm{N}: 0$ sample, as shown in Fig. 5. This slight difference may be due to differences in real-time discharge currents. The real-time current was approximately $12 \mathrm{~mA}$ when the N:0 sample was analyzed, whereas it was approximately $26 \mathrm{~mA}$ for the $4: 1$ mixture. The higher current enhances both sputtering efficiency and ionization efficiency. Actually, the Eu-to-Cu IBR of the 4:1 sample was also approximately $15 \%$ higher than that of the $\mathrm{N}: 0$ sample; hence, the Pr-to-Eu IBR of the 4:1 sample was close to that of the N:0 sample. In other words, the values measured in the pulsed mode can be calibrated using RSFs measured with $\mathrm{N}: 0$ calibration samples possessing the same parameters as the $4: 1$ sample.

Validating and comparing the mixing and secondary-cathode methods. GBW02902 europium oxide CRMs containing 14 rareearth impurities were used to validate the two methods, with the optimal mass ratio of 4:1 used in the continuous de mode with the mixing method. The results calibrated using RSFs determined at $35 \mathrm{~mA}, 45 \mathrm{~mA}$, and $55 \mathrm{~mA}$, as well as the standard RSFs provided by the spectrometer manufacturer, are summarized in Table 3, with the average values of six replicates and their standard deviations (SDs) reported. The SDs were obtained by combining the IBR and RSF RSDs. Relative deviations $(D / \%)$ were calculated using the equation: $D=\left(C_{\text {measured }}-C_{\text {certified }}\right) / C_{\text {certified }} \times 100 \%$. Table 3 reveals that the results calibrated using RSFs at $35 \mathrm{~mA}$ best match the certified values, with relative deviations of less than $15 \%$ for most oxides; relative deviations of $30 \%$ and $45 \%$ were determined at 45 $\mathrm{mA}$ and $55 \mathrm{~mA}$, respectively, which is ascribable to the matrix effect. It is noteworthy that the results calibrated using the StdRSFs are in good agreement with the certified values, with relative deviations of less than $20 \%$. Therefore, from the perspective of simplicity, calibration using the StdRSFs is greatly advantageous when an accuracy of $\pm 20 \%$ is acceptable. 
Table 3. Data Acquired in the Continuous DC Mode Using the Mixing Method with Certified Values

\begin{tabular}{|c|c|c|c|c|c|c|c|c|c|}
\hline \multirow[t]{2}{*}{ Oxide } & \multicolumn{5}{|c|}{ Mass fraction (mg/kg) ${ }^{[a]}$} & \multirow[t]{2}{*}{$\mathrm{D}_{1}{ }^{[\mathrm{cl}]} / \%$} & \multirow[t]{2}{*}{$\mathrm{D}_{2}^{[\mathrm{c}]} / \%$} & \multirow[t]{2}{*}{$\mathrm{D}_{3}^{[\mathrm{cl}] \%}$} & \multirow[t]{2}{*}{$\mathbf{D}_{4}^{[\mathrm{c}]} / \%$} \\
\hline & Certified & RSF-35 mA $A^{[b]}$ & RSF-45 mA ${ }^{[\mathrm{b}]}$ & RSF-55 mA ${ }^{[\mathrm{b}]}$ & StdRSF $^{[b]}$ & & & & \\
\hline $\mathrm{Y}_{2} \mathrm{O}_{3}$ & $17.2 \pm 1.0$ & $16.9 \pm 2.4$ & $20.7 \pm 2.9$ & $24.1 \pm 3.4$ & 16.2 & -1.5 & 20.1 & 40.2 & -5.9 \\
\hline $\mathrm{La}_{2} \mathrm{O}_{3}$ & $12.8 \pm 1.2$ & $14.3 \pm 2.0$ & $15.3 \pm 2.2$ & $16.5 \pm 2.4$ & 10.5 & 12.1 & 19.3 & 29.2 & -17.6 \\
\hline $\mathrm{CeO}_{2}$ & $3.4 \pm 0.4$ & $3.9 \pm 0.5$ & $4.1 \pm 0.5$ & $4.2 \pm 0.5$ & 2.8 & 15.0 & 19.7 & 23.9 & -16.8 \\
\hline $\operatorname{Pr}_{6} \mathbf{O}_{11}$ & $15.2 \pm 1.5$ & $12.3 \pm 1.8$ & $13.5 \pm 2.0$ & $17.1 \pm 2.5$ & 15.6 & -19.1 & -11.1 & 12.2 & 2.9 \\
\hline $\mathrm{Nd}_{2} \mathrm{O}_{3}$ & $11.8 \pm 0.6$ & $10.2 \pm 1.4$ & $11.3 \pm 1.6$ & $13.0 \pm 1.8$ & 9.8 & -13.8 & -4.2 & 10.6 & -16.7 \\
\hline $\mathrm{Sm}_{2} \mathrm{O}_{3}$ & $15.3 \pm 1.8$ & $15.2 \pm 2.2$ & $16.6 \pm 2.4$ & $18.8 \pm 2.7$ & 13.2 & -0.9 & 8.4 & 23.1 & -13.6 \\
\hline $\mathbf{G d}_{2} \mathbf{O}_{3}$ & $16.8 \pm 1.3$ & $16.2 \pm 2.1$ & $18.2 \pm 2.3$ & $22.6 \pm 2.9$ & 15.0 & -3.5 & 8.5 & 34.2 & -10.9 \\
\hline $\mathbf{T b}_{4} \mathbf{O}_{7}$ & $12.2 \pm 1.0$ & $11.3 \pm 1.3$ & $12.5 \pm 1.4$ & $15.6 \pm 1.8$ & 10.8 & -7.3 & 2.5 & 27.8 & -11.5 \\
\hline $\mathrm{Dy}_{2} \mathrm{O}_{3}$ & $11.3 \pm 0.6$ & $11.1 \pm 1.0$ & $12.2 \pm 1.1$ & $13.5 \pm 1.2$ & 10.2 & -2.2 & 7.7 & 19.0 & -10.1 \\
\hline $\mathrm{Ho}_{2} \mathrm{O}_{3}$ & $15.0 \pm 1.0$ & $13.3 \pm 1.3$ & $14.9 \pm 1.4$ & $18.3 \pm 1.8$ & 13.9 & -11.0 & -0.6 & 21.8 & -7.5 \\
\hline $\mathrm{Er}_{2} \mathrm{O}_{3}$ & $12.6 \pm 1.1$ & $12.6 \pm 1.2$ & $13.9 \pm 1.3$ & $15.6 \pm 1.5$ & 12.2 & 0.3 & 10.7 & 24.0 & -2.8 \\
\hline $\mathbf{T m}_{2} \mathbf{O}_{3}$ & $10.2 \pm 0.7$ & $10.3 \pm 0.8$ & $11.5 \pm 0.9$ & $13.7 \pm 1.1$ & 9.2 & 1.3 & 12.9 & 34.4 & -9.8 \\
\hline $\mathrm{Yb}_{2} \mathrm{O}_{3}$ & $16.3 \pm 0.6$ & $18.6 \pm 1.5$ & $20.8 \pm 1.6$ & $22.1 \pm 1.7$ & 13.2 & 14.0 & 27.5 & 35.5 & -18.8 \\
\hline $\mathbf{L u}_{2} \mathbf{O}_{3}$ & $11.6 \pm 0.8$ & $11.7 \pm 0.7$ & $12.7 \pm 0.7$ & $14.7 \pm 0.8$ & 11.0 & 1.1 & 9.5 & 26.7 & -5.3 \\
\hline
\end{tabular}

${ }^{[a]}$ Mean $\pm \mathrm{SD}(\mathrm{n}=6) ;{ }^{[b]}$ measured values calibrated using RSFs at different discharge currents; ${ }^{[\mathrm{cc}]} \mathrm{D}_{1}, \mathrm{D}_{2}, \mathrm{D}_{3}$, and $\mathrm{D}_{4}$ are relative deviations between certified and measured values calibrated using RSFs at $35 \mathrm{~mA}, 45 \mathrm{~mA}$, and $55 \mathrm{~mA}$, and StdRSFs, respectively.

Table 4. Data Acquired in the Microsecond-pulsed Mode Using the Mixing and Secondary-cathode Methods with Certified Values

\begin{tabular}{|c|c|c|c|c|c|c|c|c|c|}
\hline \multirow[t]{2}{*}{ Oxide } & \multicolumn{5}{|c|}{$\operatorname{Mass}$ fraction(mg/kg $)^{[a]}$} & \multirow[t]{2}{*}{$\mathbf{D}_{\mathrm{M1}}{ }^{[\mathrm{d}]} / \%$} & \multirow[t]{2}{*}{$\mathbf{D}_{\mathrm{M} 2}[\mathrm{~d}] / \%$} & \multirow[t]{2}{*}{$\mathrm{D}_{\mathrm{S} 1}{ }^{[\mathrm{e}]} / \%$} & \multirow[t]{2}{*}{$\mathrm{D}_{\mathrm{S} 2}[\mathrm{e}] / \%$} \\
\hline & Certified & M-RSF ${ }^{[b]}$ & M-StdRSF $^{[\mathrm{b}]}$ & S-RSF ${ }^{[\mathrm{c}]}$ & S-StdRSF $^{[\mathrm{c}]}$ & & & & \\
\hline $\mathbf{Y}_{2} \mathbf{O}_{3}$ & $17.2 \pm 1.0$ & $17.1 \pm 1.6$ & 20.7 & $17.9 \pm 1.7$ & 23.4 & -0.4 & 20.3 & 4.3 & 36.0 \\
\hline $\mathrm{La}_{2} \mathrm{O}_{3}$ & $12.8 \pm 1.2$ & $13.8 \pm 1.3$ & 18.8 & $13.7 \pm 1.3$ & 16.7 & 7.5 & 47.1 & 6.8 & 30.6 \\
\hline $\mathrm{CeO}_{2}$ & $3.4 \pm 0.4$ & $4.1 \pm 0.4$ & 3.9 & $4.4 \pm 0.4$ & 4.2 & 19.2 & 15.4 & 30.0 & 22.3 \\
\hline $\operatorname{Pr}_{6} \mathrm{O}_{11}$ & $15.2 \pm 1.5$ & $15.1 \pm 1.5$ & 15.9 & $16.4 \pm 1.6$ & 17.5 & -0.3 & 4.9 & 7.8 & 15.1 \\
\hline $\mathrm{Nd}_{2} \mathrm{O}_{3}$ & $11.8 \pm 0.6$ & $12.8 \pm 1.2$ & 15.4 & $13.8 \pm 1.3$ & 17.2 & 8.7 & 30.2 & 16.8 & 45.5 \\
\hline $\mathrm{Sm}_{2} \mathrm{O}_{3}$ & $15.3 \pm 1.8$ & $16.8 \pm 1.6$ & 19.2 & $17.7 \pm 1.7$ & 19.2 & 9.7 & 25.3 & 15.7 & 25.3 \\
\hline $\mathbf{G d}_{2} \mathrm{O}_{3}$ & $16.8 \pm 1.3$ & $17.2 \pm 1.5$ & 21.2 & $19.1 \pm 1.7$ & 27.0 & 2.5 & 25.9 & 13.9 & 60.9 \\
\hline $\mathbf{T b}_{\mathbf{4}} \mathbf{O}_{7}$ & $12.2 \pm 1.0$ & $12.1 \pm 0.9$ & 13.7 & $13.5 \pm 1.0$ & 16.2 & -0.6 & 12.0 & 10.9 & 32.8 \\
\hline $\mathrm{Dy}_{2} \mathrm{O}_{3}$ & $11.3 \pm 0.6$ & $11.0 \pm 0.7$ & 14.1 & $12.4 \pm 0.7$ & 15.8 & -2.5 & 24.5 & 9.7 & 40.2 \\
\hline $\mathrm{Ho}_{2} \mathrm{O}_{3}$ & $15.0 \pm 1.0$ & $13.2 \pm 0.9$ & 17.8 & $14.5 \pm 1.0$ & 20.1 & -11.8 & 18.8 & -3.5 & 34.1 \\
\hline $\mathrm{Er}_{2} \mathrm{O}_{3}$ & $12.6 \pm 1.1$ & $12.4 \pm 0.8$ & 14.0 & $13.6 \pm 0.9$ & 16.1 & -1.5 & 11.0 & 8.0 & 27.9 \\
\hline $\mathbf{T m}_{2} \mathbf{O}_{3}$ & $10.2 \pm 0.7$ & $9.9 \pm 0.5$ & 12.2 & $10.5 \pm 0.6$ & 13.5 & -2.8 & 20.1 & 2.9 & 32.6 \\
\hline $\mathbf{Y} \mathbf{b}_{2} \mathbf{O}_{3}$ & $16.3 \pm 0.6$ & $18.7 \pm 1.0$ & 22.1 & $17.9 \pm 1.0$ & 15.8 & 14.5 & 35.6 & 9.9 & -3.0 \\
\hline $\mathbf{L u}_{\mathbf{2}} \mathrm{O}_{\mathbf{3}}$ & $11.6 \pm 0.8$ & $10.3 \pm 0.4$ & 13.3 & $11.7 \pm 0.4$ & 15.8 & -11.0 & 15.0 & 0.6 & 36.2 \\
\hline
\end{tabular}

${ }^{[a]}$ Mean $\pm \mathrm{SD}(\mathrm{n}=6) ;{ }^{[b]}$ measured values using the mixing method calibrated using the RSFs in this work and StdRSFs, respectively; ${ }^{[\mathrm{c}]}$ measured values using the secondary-cathode method calibrated using RSFs in this work and StdRSFs, respectively; ${ }^{[\mathrm{d}]} \mathrm{D}_{\mathrm{M} 1}$ and $\mathrm{D}_{\mathrm{M} 2}$ are relative deviations between certified and measured values using the mixing method calibrated using RSFs in this work and StdRSFs, respectively; ${ }^{\left[{ }^{[}\right]} \mathrm{D}_{\mathrm{S} 1}$ and $\mathrm{D}_{\mathrm{S} 2}$ are relative deviations between certified and measured values using the secondary-cathode method calibrated using RSFs in this work and StdRSFs, respectively.

Table 5. Mass Fractions (mean \pm 2SD) of Rare-earth Oxides in a Europium Oxide Sample, $\mathrm{mg} / \mathrm{kg}$

\begin{tabular}{|c|c|c|c|}
\hline Oxide & Mass fraction & Oxide & Mass fraction \\
\hline $\mathbf{Y}_{2} \mathbf{O}_{3}$ & $1.7 \pm 0.5$ & $\mathbf{T b}_{4} \mathbf{O}_{7}$ & $<0.001$ \\
\hline $\mathbf{L a}_{2} \mathbf{O}_{3}$ & $<0.002$ & $\mathrm{Dy}_{2} \mathrm{O}_{3}$ & $<0.001$ \\
\hline $\mathrm{CeO}_{2}$ & $0.40 \pm 0.16$ & $\mathrm{Ho}_{2} \mathrm{O}_{3}$ & $<0.004$ \\
\hline $\operatorname{Pr}_{6} \mathbf{O}_{11}$ & $3.1 \pm 0.7$ & $\mathrm{Er}_{2} \mathrm{O}_{3}$ & $0.23 \pm 0.09$ \\
\hline $\mathrm{Nd}_{2} \mathrm{O}_{3}$ & $2.5 \pm 0.5$ & $\mathbf{T m}_{2} \mathbf{O}_{3}$ & $<0.001$ \\
\hline $\mathrm{Sm}_{2} \mathrm{O}_{3}$ & $7.9 \pm 2.4$ & $\mathbf{Y} \mathbf{b}_{2} \mathbf{O}_{3}$ & $<0.019$ \\
\hline $\mathbf{G d}_{2} \mathbf{O}_{3}$ & $3.4 \pm 1.3$ & $\mathbf{L u}_{2} \mathbf{O}_{3}$ & $0.12 \pm 0.04$ \\
\hline
\end{tabular}

Both, the mixing and secondary-cathode methods, were used in the microsecond-pulsed mode. The RSFs of the rare-earth elements in the microsecond-pulsed mode with the optimized parameters listed in Table 4 were measured using the calibration samples, the details of which are found elsewhere. ${ }^{18}$ The results obtained using the RSFs and StdRSFs were compared; the measured values calibrated using the RSFs in this work are in good agreement with the certified values for the mixing and secondary- cathode methods, with relative deviations of less than $15 \%$ determined for most elements. However, the results calibrated using the StdRSFs exhibit poor accuracies, with relative deviations of up to $40 \%$ and $50 \%$ for the mixing and secondary-cathode methods, respectively, which is probably ascribable to the fact that the StdRSFs were determined in the dc mode, rather than in the pulsed mode.

Europium oxide sample analysis. In view of its lower LoQs, the secondary-cathode method was used to analyze a europium oxide sample, with the results calibrated using the RSFs determined in this work. The results for the 14 rare-earth oxides are summarized in Table 5. The sum of the mass fractions of all rare-earth oxides was determined to be $19.3 \pm 2.9 \mathrm{mg} / \mathrm{kg}$ (mean $\pm 2 \mathrm{SD}$ ); hence the relative purity of the $\mathrm{Eu}_{2} \mathrm{O}_{3}$ sample is $99.9981 \pm 0.0003 \%$. According to the Chinese National Standard Terms of Rare Earths (GB/T 15676-2015), this $\mathrm{Eu}_{2} \mathrm{O}_{3}$ sample was confirmed to be a high-purity rare-earth oxide ( $>99.99 \%)$. 


\section{CONCLUSIONS}

We developed mixing and secondary-cathode methods for the microsecond-pulsed and continuous direct-current glow-discharge mass spectrometric analyses of trace rare-earth oxides present in europium oxide samples. A 4:1 mass ratio of high-purity copper to $\mathrm{Eu}_{2} \mathrm{O}_{3}$ was determined to be optimal for the mixing method, while a $5 \mathrm{~mm}$-diameter hole was optimal for the secondary-cathode method. LoQs of the order of $10^{-9}$ were determined for the secondary-cathode method, which are approximately one order of magnitude lower than those obtained using the mixing method. A pronounced matrix effect was observed in the continuous dc mode, but it was sufficiently low to be ignored in the microsecond-pulsed mode. The matrix effect is probably the result of real-time variations in the values of the discharge parameters in various matrix samples, and was corrected for after optimizing the discharge parameters. The accuracies of the methods were validated using $\mathrm{Eu}_{2} \mathrm{O}_{3}$ CRMs; $\mathrm{RSF}$ calibration led to good agreement with the certified values, with relative deviations of less than $15 \%$ for most elements. The results calibrated using StdRSFs were also in good agreement with the certified values when the continuous dc mode was used, with relative deviations of less than $20 \%$, whereas, StdRSF calibration in the microsecond-pulsed mode resulted in poor accuracies, with relative deviations of up to $40 \%$ and $50 \%$ for the mixing and secondary-cathode methods, respectively.

In summary, the pulsed mode is advantageous over the continuous de mode in terms of qualification limits and sample quantities because the secondary-cathode method, which exhibits lower LoQs for smaller sample amounts, can be used in this mode. On the other hand, the continuous dc mode provides superior accuracies when calibrated using StdRSFs, while the dc and pulsed modes provide similar accuracies when calibrated using the RSFs determined in this work, regardless of which method (mixing or secondary-cathode) was used.

Finally, the 14 rare-earth oxides in a $\mathrm{Eu}_{2} \mathrm{O}_{3}$ sample were determined, with the relative purity of the sample found to be $99.9981 \pm 0.0003 \%$, which meets the product standard of a highpurity rare-earth oxide.

\section{AUTHOR INFORMATION}

\section{Corresponding Author}

*T. Zhou

Email address: zhoutao@nim.ac.cn

\section{Notes}

The authors declare no competing financial interest.

\section{ACKNOWLEDGMENTS}

Financial support by Ministry of Science and Technology of the People's Republic of China (2019YFF0217101) is gratefully acknowledged.

\section{REFERENCES}

1. J. Yin, B. Hu, M. He and Z. C. Jiang, At. Spectrosc., 2005, 26, 197-202. https://doi.org/1 0.46770/AS.2005.05.006

2. W. R. Pedreira, C. A. da Silva Queiroz, A. Abrao and M. M. Pimentel, J. Alloy. Compd., 2004, 374, 129-132. https://doi.org/10.1016/j.jallcom.2003.11.148

3. X. Zhang, Y. Yi, Y. Liu, X. Li, J. Liu, Y. Jiang and Y. Su, Anal. Chim. Acta, 2006, 555, 57-62. https://doi.org/10.1016/j.aca.2005.08.055

4. V. Hoffmann, M. Kasik, P. K. Robinson and C. Venzago, Anal. Bioanal. Chem., 2005, 381, 173-188. https://doi.org/10.1007/s00216-004-2933-2

5. R. Matschat, J. Hinrichs and H. Kipphardt, Anal. Bioanal. Chem., 2006, 386, 125-141. https://doi.org/ 10.1007/s00216-006-0645-5

6. W. Hang and W. W. Harrison, Anal. Chem., 1997, 69, 4957-4963. https://doi.org/10.1021/ac9710386

7. R. Qian, S. Zhuo, Z. Wang and P. K. Robinson, J. Anal. At. Spectrom., 2013, 28, 1061-1067. https://doi.org/10.1039/C3JA50029B

8. J. Dong, R. Qian, W. Xiong, H. Qu, B. Siqin, S. Zhuo and P. K. Robinson, Int. J. Mass Spectrom., 2014, 361, 1-8. https://doi.org/10.1016/j.ijms.2014.01.018

9. W. Schelles and R. E. Van Grieken, Anal. Chem., 1996, 68, 3570-3574. https://doi.org/10.1021/ac960441u

10. W. Schelles and R. Van Grieken, Anal. Chem., 1997, 69, 2931-2934. https://doi.org/10.1021/ac970186t

11. J. C. Woo, N. Jakubowski and D. Stuewer, J. Anal. At. Spectrom., 1993, 8, 881-889. https://doi.org/10.1039/JA9930800881

12. S. Jung, S. Kim and J. Hinrichs, Spectrochim. Acta B, 2016, 122, 46-51. https://doi.org/10.1016/j.sab.2016.05.003

13. W. Vieth and J. C. Huneke, Spectrochim. Acta B, 1991, 46, 137-153. https://doi.org/10.1016/0584-8547(91)80017-W

14. T. Gusarova, T. Hofmann, H. Kipphardt, C. Venzago, R. Matschat and U. Panne, J. Anal. At. Spectrom., 2010, 25, 314-321. https://doi.org/10.1039/B921649A

15. J. Zhang, T. Zhou, Y. Tang, Y. Cui and J. Li, J. Anal. At. Spectrom., 2016, 31, 2182-2191. https://doi.org/10.1039/C6JA00172F

16. T. Gusarova, B. Methven, H. Kipphardt, R. Sturgeon, R. Matschat and U. Panne, Spectrochim. Acta B, 2011, 66, 847-854. https://doi.org/10.1016/j.sab.2011.12.001

17. J. Zhang, T. Zhou, D. Song, Y. Tang, Y. Cui and B. Wu, Spectrochim. Acta B, 2020, 164, 105748. https://doi.org/10.1016/j.sab.2019.105748

18. J. Zhang, T. Zhou, Y. Cui, Y. Tang and B. Wu, J. Anal. At. Spectrom., 2020, 35, 2712-2722. https://doi.org/10.1039/D0JA00327A. 